\title{
Sub-maxwellian source injection and negative differential transconductance in decorated graphene nanoribbons
}

\author{
Damiano Marian, ${ }^{1}$ Enrique G. Marin,,${ }^{1,2}$ Giuseppe Iannaccone,${ }^{1}$ and Gianluca Fiori ${ }^{1}$ \\ ${ }^{1}$ Dipartimento di Ingegneria dell'Informazione, Università di Pisa, 56122, Pisa, Italy \\ ${ }^{2}$ Departamento de Electrónica, Universidad de Granada, 18071, Granada, Spain
}

\begin{abstract}
The precise engineering of the graphene crystal structure at the atom level, enabled by the recent advances in synthesis approaches, has driven a renewed surge of interest in graphene nanoribbons (GNRs), whose electronic properties can be tuned by the arrangement of atoms at their edges. This technological option opens up the possibility of devising alternative devices based on carrier transport through topological states. In this work, by means of multiscale calculations we investigate field-effect transistors based on topological GNRs with shaped edges, demonstrating the possibility of obtaining large negative differential transconductance effects, beating the Boltzmann limit for thermionic injection.
\end{abstract}

\section{INTRODUCTION}

After more than 15 years, graphene keeps attracting a big part of the research interests in the field of materials science. The progresses resulting from the synergetic mix of theoretical analysis and experimental findings have opened the exploration of fields like spintronics based on magnetic configurations in zig-zag nanoribbons [1], valleytronics linked to topological states in bilayer graphene [2, 3], and, more recently, twistronics in precisely oriented graphene layers leading to superconductivity at low temperature [4].

The prospects have been sharply boosted by the technical capability to engineer the two-dimensional crystal structure at the atomic scale. The most advanced materials design is nowadays available for graphene nanoribbons, where thanks to a bottom-up synthesis driven by molecular precursors [5], it has been demonstrated that, by engineering nanoribbons with varied choreographed shapes, it is possible to, e.g. i) obtain type I and type II heterojunctions, (junctions of a small- and a large-bandgap GNR, where the bandgap of the former lies within bandgap of the latter; or junctions where the bandgaps are staggered) $[6,7], i i)$ create individual 1D channels by destructive quantum interference in nanoporous structures [8,9] or iii) devise topological states at junctions between nanoribbons of different width $[10,11]$. In spite of the variety of breakthrough experimental findings and of the theoretical understanding at the materials level, it is not evident how to exploit these electrical properties at the device level.

In this work we investigate a particular family of GNRs with choreographed width. To this purpose, we combine tight-binding calculations of the bandstructure, that have been demonstrated to be in excellent agreement with the experimental observations [10, 11], and quantum transport simulations within the framework of Non-Equilibrium Green Functions [12] that are selfconsistently solved with the 3D Poisson equation, in order to determine the device performance in the ballistic regime. The particular shape of the GNR bandstructures results in the observation of two effects of interest: $i$ ) a pronounced negative differential transconductance, and ii) a current modulation below the Boltzmann limit at room temperature.

\section{ENGINEERED GRAPHENE NANORIBBONS}

Engineering the edges of a GNR introduces many degrees of freedom in the design space, since edge properties directly alter GNR electronic properties. Amongst the several possibilities, we have opted for two families of armchair GNRs (AGNRs). Specifically, we have considered $N$-AGNRs, being $N$ the number of atoms in the GNR transverse direction, that are extended in a symmetric (also called in-line) or asymmetric (staggered) arrangements, to $(N+4)$ atoms in the case of the in-line structure or $(N+2)$ atoms for the staggered one; as in Figure 1(a) and and (d), where the pristine backbone GNR is shown in blue, while the decoration is highlighted with transparent red lines.

The length of the wider GNR segments and their distance unambiguously determine the structure. In particular, we use a double index notation $(n, m)$, according to Ref. [11] , with $n$ specifying the length of the wider segment, while the index $m$ specifies the distance between two successive wider segments. The indexes $n$ and $m$ are counted in integers of the bare AGNR unitary cell, i.e. $n \cdot 3 a_{\mathrm{cc}}$ and $m \cdot 3 a_{\mathrm{cc}}$, where $a_{\mathrm{cc}}$ is the carbon-carbon distance, (see Figure 1(a) and (d).

To the purpose of device design, among a broad range of available options, we have opted for $N=7$ atoms. We have considered $m=2$, fixing the distance between extended segments, while we have varied their length from $n=3$ up to $n=8$ (see Figure 1(a) and (d). The resulting band-structures, as calculated from tight-binding (TB) model, are shown in Figure 1(b) and (e). The TB calculations have been shown to reproduce the actual bandstructure obtained from ab-initio simulations and experimental results $[10,11]$. The TB Hamiltonian, following Ref. [11], is a $\mathrm{p}_{z}$ orbital nearest neighbour model with a hopping parameter $t=3.0 \mathrm{eV}$. As can be seen, this selection of $N, n$ and $m$ is already representative of really different electronic behaviours, depending on the GNR shape. In particular, in-line and staggered geometries 

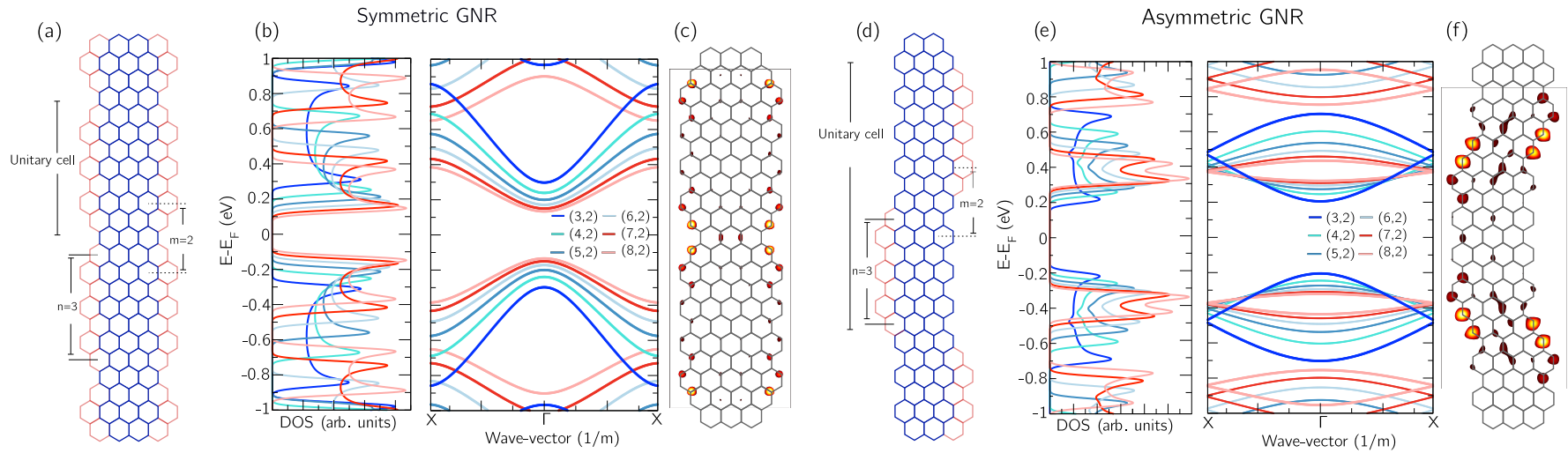

FIG. 1. Schematic choreographed 7-AGNR, 7 being the number of C atoms of the pristine GNR in the transverse direction, in the (a) in-line and (d) staggered configurations, also exemplifying the double index notation $(n, m)$ in the case $(3,2)$. Density of states and bandstructure of different GNRs calculated from the TB Hamiltonian with $m=2$ and $n$ varying from 3 to 8 for the (b) in-line and (e) staggered configurations. (c) Projected density of states of the first quantized level in the conduction band for a $(4,2)(\mathrm{c})$ in-line and (f) staggered GNR.

show opposite behaviour of the bandgap with increasing $n$, i.e. decreasing from $0.58 \mathrm{eV}$ for $n=3$ to $0.26 \mathrm{eV}$ for $n=8$ in the in-line structures, and increasing from 0.41 $\mathrm{eV}$ for $n=3$ to $0.64 \mathrm{eV}$ for $n=8$ in the staggered structures. The effective mass at the $\Gamma$ point shows a similar trend, decreasing from $0.30 m_{0}$ for $n=3$ to $0.13 m_{0}$ for $n=8$ for in-line GNRs and increasing from $0.26 m_{0}$ for $n=3$ to $0.41 m_{0}$ for $n=8$ for staggered structures, with $m_{0}$ the electron mass. The associated density of states, calculated using a Gaussian smearing function with $\sigma=25.9 \mathrm{meV}$, is plotted in Figure 1(b) and (e) showing a non-monotonic trend (with pronounced valleys) that is steeper as $n$ is increased. More importantly, the states defining the valence and conduction bands are localized at the atoms that define the transition between different segments (Figure 1(c) and (f) and, with the exception of the staggered $(n, m)=(3,2)$, are topological, as it has been demonstrated, using a topological invariant based on Zak phases, in [11]. The longitudinal distance between the atoms defining the topological states, i.e the ones yellow-coloured in the projected density of states in Figure $1(\mathrm{c})$ and (f), vary from $19.88 \AA$ in the staggered nanoribbon to $2.84 \AA$ in the in-line GNR.

\section{TOPOLOGICAL DEVICES}

Here, we study the performance of topological devices, exploiting the decorated GNR discussed in the previous section as channel material. The topological states are expected to command the device operation, being the space localization and energy quantization associated to these states determinant for the electronic transport. To the purpose of analysing this aspect, the TB Hamiltonian is used in the NanoTCAD ViDES suite $[13,14]$, where quantum transport and electrostatics are self-consistently solved, at room temperature, i.e. $T=300 \mathrm{~K}$. As shown in Figure 2 we have considered a double-gate field-effect transistor (FET) where $L_{\mathrm{ch}}$ and
$L_{\mathrm{S} / \mathrm{D}}$ are the channel and source/drain lengths, respectively. The gates are isolated from the GNR by top and bottom dielectric layers with equivalent silicon oxide thickness $t_{\mathrm{ox}}=0.5 \mathrm{~nm}$. The source and drain are heavily doped with donors, in order to align the Fermi level with the middle energy of the conduction band; e.g. for the $(4,2)$ in-line GNR the energy degeneracy at source/drain is $0.4 \mathrm{eV}$. Given the symmetric nature of the bands, equivalent results can be expected for hole transport.

First, we have investigated the transfer characteristics, i.e., drain current, $I_{\mathrm{DS}}$, vs. gate voltage $V_{\mathrm{GS}}$, of several inline and staggered GNRs with different $n$, i.e., $n=3 \ldots 8$ (as shown in Fig. 3) for a drain-to-source voltage $V_{\mathrm{DS}}=$ $0.1 \mathrm{~V}$. The channel and source/drain lengths are an integer multiple of the GNR unit cell, resulting in channel and source/drain lengths varying with $n=3 \cdots 8$ from $L_{\mathrm{ch}} / L_{\mathrm{S} / \mathrm{D}}=6.8 \mathrm{~nm}$ up to $L_{\mathrm{ch}} / L_{\mathrm{S} / \mathrm{D}}=15.3 \mathrm{~nm}$. For a fair comparison, we have assumed the same device lengths for both the in-line and the staggered GNRs.

For both types of nanoribbons, a sharp negative differential transconductance (NDTC) effect can be observed.

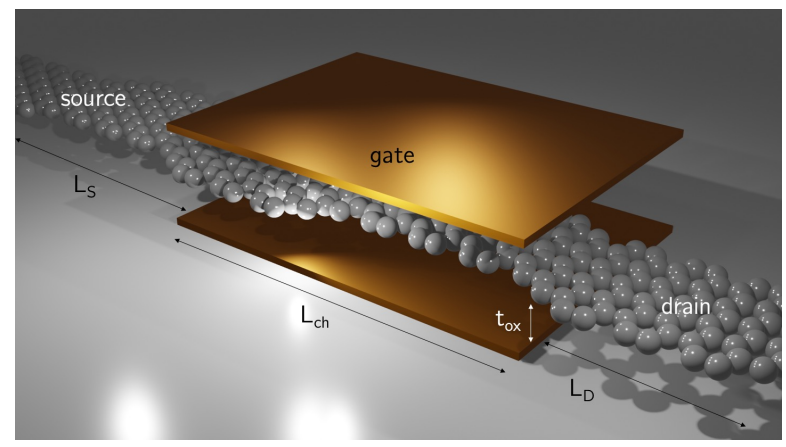

FIG. 2. Schematic of the double-gate FET with a decorated GNR as channel. $t_{\mathrm{ox}}, L_{\mathrm{ch}}$ and $L_{\mathrm{S} / \mathrm{D}}$ are the equivalent oxide thickness, channel length, and source/drain length, respectively. 

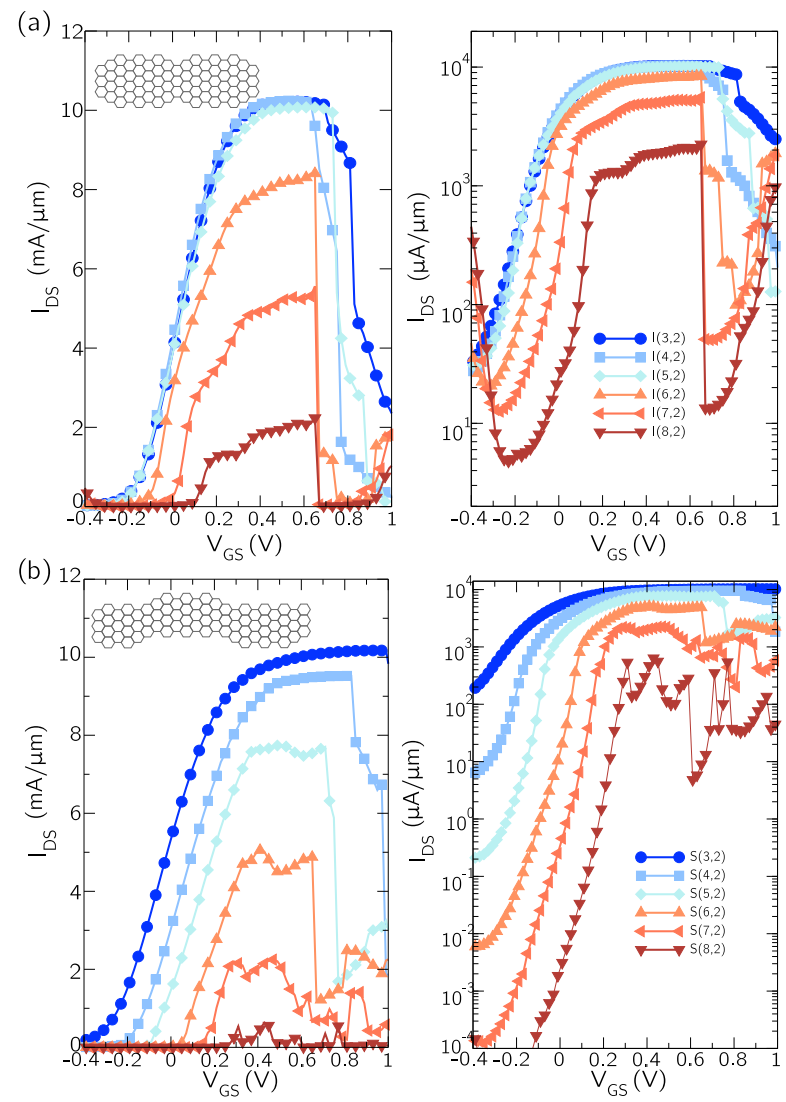

FIG. 3. Transfer characteristics in linear (left) and semilogarithmic (right) scale of the in-line (top) and staggered (bottom) GNR FETs for $V_{\mathrm{DS}}=0.1 \mathrm{~V}$.

The NDTC is motivated by the strong energy quantization of the GNRs bandstructure - discussed later in more detail from the local density of states (Figure 4)-, with a transport mechanism associated to the topological states localized at the transitions between different segments of the GNR, that enable large current modulations.

In particular for the in-line structures, the peak-tovalley ratio is as large as two orders of magnitude for $n=8$ reducing to a factor of 5 for $n=3$. The NDTC transition is steep, occurring in a few tens of $\mathrm{mV}$ for $n=6-8$, with calculated slopes of a $11 \mathrm{mV}$ per decade for $n=7$ and 8 in-line GNRs. Additionally, the conductive peak is widened as $n$ is reduced, corresponding to the broader DOS associated to the conduction band in these structures (Figure 1(b) and (e). The onset of the p-type conduction can also be observed (in the semilogarithmic plot) for higher values of $n$ in the in-line structures, driven by inter-band tunnelling mechanisms.

The NDTC effect is a consequence of the misalignment of the high DOS peaks at the channel and at the source, being the energy of the former shifted with $V_{\mathrm{GS}}$. This argument is discussed in Figure 4 where (a) the local density of states (LDOS) is plotted as a function of the longitudinal position, and b) the current spectrum is plotted vs. energy. Both magnitudes are shown for the $(6,2)$ in-line GNR, and two different gate voltages before and after the NDTC transition. First, a strong quantization of the DOS appears in the channel as a consequence of the spatial confinement, leading to a sharp change in the LDOS available within the transport window. As $V_{\mathrm{GS}}$ is increased from $0.6 \mathrm{~V}$ to $0.8 \mathrm{~V}$, the quantized LDOS is suppressed in the transport window, as can be seen in the LDOS, and, as a consequence, the current spectrum falls abruptly in this energy range (Figure 4(b), directly impacting in the $I_{\mathrm{DS}}$.

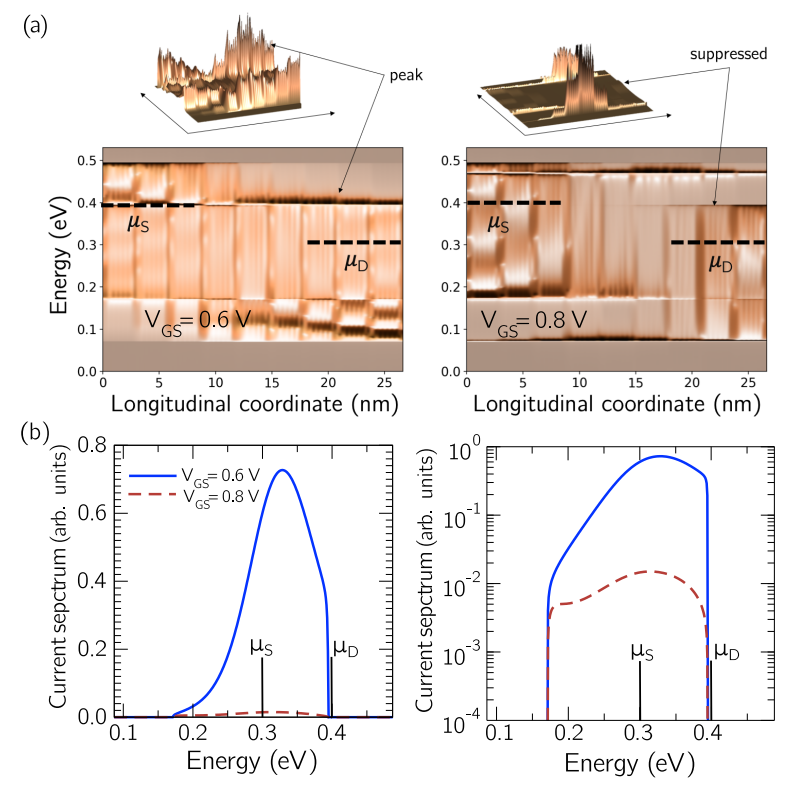

FIG. 4. (a) Local density of states (LDOS) as a function of the energy and longitudinal position, and (b) Current spectrum in linear and semi-logarithmic scales, for two $V_{\mathrm{GS}}$ bias close to the NDC transition for the in-line $(n, m)=(6,2)$ structure. The Fermi level at the source, $\mu_{s}$, and at the drain, $\mu_{d}$, are marked in both figures.

In light of the strong impact that the sharp and non-monotonic transitions in the DOS have on the ON state transfer characteristic, we have further analysed the possibility to use the GNR band-structure to obtain a steep OFF-ON switching of the FET exploring different channel lengths. In particular, we explore the potential to reach a sub-maxwellian sub-threshold swing (SS), defined as the inverse of the slope of the $\log (I)-V_{\mathrm{G}}$ curves, through DOS limited injection, therefore seeking low power operation. This proposal, that utilizes a sharp density of states as a cold source, has been very recently introduced as a way to beat the Boltzmann limit of $60 \mathrm{mV} / \mathrm{dec}$ at room temperature in FETs based on carbon nanotubes [15], $\mathrm{MoS}_{2}[16,17]$ and lateral heterostructures of 2D materials [18], but, as we will show, this effect is more pronounced in the proposed devices. In order to observe it in GNRs, we have chosen $(n, m)=(4,2)$, varying the channel length from $\sim 5 \mathrm{~nm}$ up to $\sim 17 \mathrm{~nm}$, so that the tunnel current in the OFF state is eventually suppressed.

Figure 5 shows the obtained transfer characteristics in semilogarithmic scale for $V_{\mathrm{DS}}=0.1 \mathrm{~V}$. As can be 

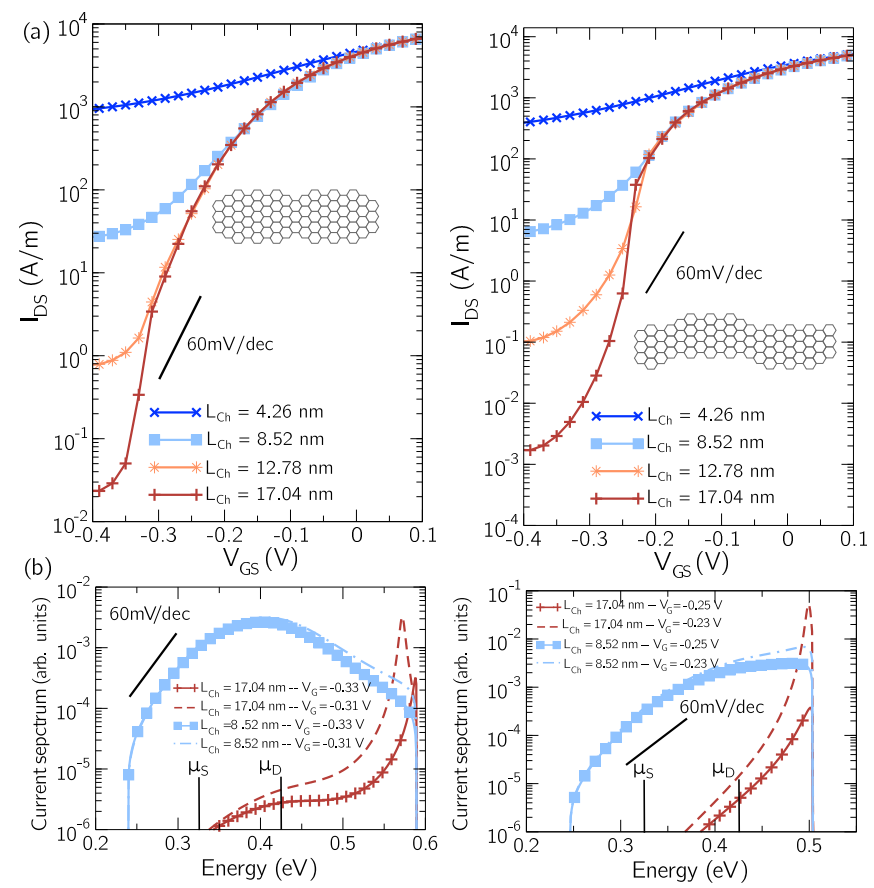

FIG. 5. (a) Transfer characteristics in semilogarithmic scale for the in-line (left) and staggered (right) GNRs FETs for different channel lengths for $V_{\mathrm{DS}}=0.1 \mathrm{~V}$. The $60 \mathrm{mV} / \mathrm{dec}$ Boltzmann limit is also shown. (b) Current spectrum for two channel lengths $\left(L_{\mathrm{ch}}=8.52 \mathrm{~nm}\right.$ and $\left.L_{\mathrm{ch}}=17.04 \mathrm{~nm}\right)$ and two gate voltages in the sub-threshold region in the in-line and staggered structures.

seen, $S S$ is reduced as $L_{\mathrm{ch}}$ is increased with the suppression of the intra-band tunneling. When injection is not dominated by tunneling, $S S$ can be as small as $11 \mathrm{mV} / \operatorname{dec}(23 \mathrm{mV} / \mathrm{dec})$ for the staggered (in-line) structures with $L_{\mathrm{ch}}=17.04 \mathrm{~nm}$ and $26 \mathrm{mV} / \mathrm{dec}(53 \mathrm{mV} / \mathrm{dec})$ for $L_{\mathrm{ch}}=12.78 \mathrm{~nm}$. This effect is due to the sharp variation of the DOS associated to the choreographed GNRs that is able to boost the current over the Fermi distribution tail (that constrains $S S$ to the Boltzmann limit) and to the gate control of the channel potential barrier. The mechanism is more clearly appreciated in Figure 5(b) considering the current spectrum vs. energy, shown for two channel lengths $\left(L_{\mathrm{ch}}=8.52 \mathrm{~nm}\right.$ and $L_{\mathrm{ch}}=17.04 \mathrm{~nm}$ ) and two gate voltages in the in-line and staggered structures. It is clear the steep change in both configurations for the longer channel, where tunneling is suppressed, with a high peak just at the border of the conduction band channel barrier, which is close to $0.55 \mathrm{eV}(0.50 \mathrm{eV})$ in the in-line (staggered) longer devices. For the shorter channels, instead, the trend is clearly tunnel-dominated and softened by the transport energy window, with $\mu_{s}=0.425 \mathrm{eV}$ and $\mu_{d}=0.325 \mathrm{eV}$, undermining the observation of an $\mathrm{SS}<60 \mathrm{mV} / \mathrm{dec}$.

\section{CONCLUSION}

FETs using as channel GNRs with choreographed edges have been analysed by means of self-consistent electrostatic and quantum transport simulations. The exploitation of a portion of the whole configuration space shows that the strong quantization of the topological states (associated to those atoms localized at the transition between GNRs of different width) leads to promising features in the device characteristics. We have observed that a strong negative differential transconductance (NDTC), with peak-to-valley ratios ranging from $\sim 5$ up to $\sim 100$ can be achieved. The edge structure is relevant to device operation being the NDTC effect more prominent for in-line, i.e. symmetrically patterned GNRs. Additionally, the DOS quantization enables a sub-maxwellian distribution of thermionically injected charge carries leading to an $S S$ of the current-voltage characteristics as small as $11 \mathrm{mV} /$ dec for staggered structure and $23 \mathrm{mV} / \mathrm{dec}$ for the in-line structure.

\section{ACKNOWLEDGMENTS}

We gratefully acknowledge the support from the European Commission through the Graphene Flagship Core 3 (Contract No. 881603), through the Quantum Engineering for Machine Learning (QUEFORMAL) H2020 Project (Contract No. 829035) and through the Origami electronics for three-dimensional integration of computational devices (ORIGENAL) H2020 Project (Contract No. 863258). E.G.M. also acknowledges Juan de la Cierva Incorporacion IJCI-2017-32297 [Ministry of Economic Affairs and Digital Transformation (MINECO)State Research Agency (AEI)]. The authors also acknowledge support from the Italian Ministry of University and Research through the PRIN project FIVE2D (Contract No. 2017SRYEJH_001).
[1] Young Woo Son, Marvin L. Cohen, and Steven G. Louie, "Half-metallic graphene nanoribbons," Nature 444, 347349 (2006), 0611600 [cond-mat].

[2] Jing Li, Ke Wang, Kenton J. McFaul, Zachary Zern, Yafei Ren, Kenji Watanabe, Takashi Taniguchi, Zhenhua Qiao, and Jun Zhu, "Gate-controlled topological conducting channels in bilayer graphene," Nature Nanotechnology 11, 1060-1065 (2016), 1509.03912.

[3] Long Ju, Zhiwen Shi, Nityan Nair, Yinchuan Lv, Chenhao Jin, Jairo Velasco, Claudia Ojeda-Aristizabal,
Hans A. Bechtel, Michael C. Martin, Alex Zettl, James Analytis, and Feng Wang, "Topological valley transport at bilayer graphene domain walls," Nature 520, 650-655 (2015).

[4] Yuan Cao, Valla Fatemi, Shiang Fang, Kenji Watanabe, Takashi Taniguchi, Efthimios Kaxiras, and Pablo JarilloHerrero, "Unconventional superconductivity in magicangle graphene superlattices," Nature 556, 43-50 (2018).

[5] Jinming Cai, Pascal Ruffieux, Rached Jaafar, Marco Bieri, Thomas Braun, Stephan Blankenburg, Matthias 
Muoth, Ari P. Seitsonen, Moussa Saleh, Xinliang Feng, Klaus Müllen, and Roman Fasel, "Atomically precise bottom-up fabrication of graphene nanoribbons," Nature 466, 470-473 (2010).

[6] Jinming Cai, Carlo A. Pignedoli, Leopold Talirz, Pascal Ruffieux, Hajo Söde, Liangbo Liang, Vincent Meunier, Reinhard Berger, Rongjin Li, Xinliang Feng, Klaus Müllen, and Roman Fasel, "Graphene nanoribbon heterojunctions," Nature Nanotechnology 9, 896900 (2014).

[7] Yen Chia Chen, Ting Cao, Chen Chen, Zahra Pedramrazi, Danny Haberer, Dimas G. De Oteyza, Felix R. Fischer, Steven G. Louie, and Michael F. Crommie, "Molecular bandgap engineering of bottom-up synthesized graphene nanoribbon heterojunctions," Nature Nanotechnology 10, 156-160 (2015).

[8] César Moreno, Manuel Vilas-Varela, Bernhard Kretz, Aran Garcia-Lekue, Marius V. Costache, Markos Paradinas, Mirko Panighel, Gustavo Ceballos, Sergio O. Valenzuela, Diego Peña, and Aitor Mugarza, "Bottom-up synthesis of multifunctional nanoporous graphene," Science 360, 199-203 (2018).

[9] Gaetano Calogero, Isaac Alcón, Nick Papior, Antti-Pekka Jauho, and Mads Brandbyge, "Quantum interference engineering of nanoporous graphene for carbon nanocircuitry," Journal of the American Chemical Society 141, 13081-13088 (2019).

[10] Daniel J. Rizzo, Gregory Veber, Ting Cao, Christopher Bronner, Ting Chen, Fangzhou Zhao, Henry Rodriguez, Steven G. Louie, Michael F. Crommie, and Felix R. Fischer, "Topological band engineering of graphene nanoribbons," Nature 560, 204-208 (2018), 1805.06470.

[11] Oliver Gröning, Shiyong Wang, Xuelin Yao, Carlo A. Pignedoli, Gabriela Borin Barin, Colin Daniels, Andrew Cupo, Vincent Meunier, Xinliang Feng, Akimitsu Narita,
Klaus Müllen, Pascal Ruffieux, and Roman Fasel, "Engineering of robust topological quantum phases in graphene nanoribbons," Nature 560, 209-213 (2018), 1805.06635.

[12] Supriyo Datta, "Nanoscale device modeling: The green's function method," Superlattices Microstruct. 28, 253 278 (2000).

[13] S. Bruzzone, G. Iannaccone, N. Marzari, and G. Fiori, "An open-source multiscale framework for the simulation of nanoscale devices," IEEE Trans. Electron Devices 61, 48-53 (2014).

[14] "NanoTCAD ViDES," Available at: http://vides.nanotcad.com/vides/, accessed: 05-202019.

[15] Chenguang Qiu, Fei Liu, Lin Xu, Bing Deng, Mengmeng Xiao, Jia Si, Li Lin, Zhiyong Zhang, Jian Wang, Hong Guo, Hailin Peng, and Lian Mao Peng, "Diracsource field-effect transistors as energy-efficient, highperformance electronic switches," Science 361, 387-392 (2018).

[16] Fei Liu, Chenguang Qiu, Zhiyong Zhang, Lian Mao Peng, Jian Wang, and Hong Guo, "Dirac Electrons at the Source: Breaking the $60-\mathrm{mV} /$ Decade Switching Limit," IEEE Transactions on Electron Devices 65, 2736-2743 (2018).

[17] Demetrio Logoteta, Marco G Pala, Jean Choukroun, Philippe Dollfus, and Giuseppe Iannaccone, "A steepslope $\operatorname{mos}_{2}$-nanoribbon mosfet based on an intrinsic coldcontact effect," IEEE Electron Device Lett. 40, 15501553 (2019).

[18] Enrique G Marin, Damiano Marian, Marta Perucchini, Gianluca Fiori, and Giuseppe Iannaccone, "Lateral Heterostructure Field-Effect Transistors Based on 2DMaterial Stacks with Varying Thickness and Energy Filtering Source," ACS Nano 14, 1982-1989 (2020), 2001.03139 . 